\title{
Las emociones y la explicación de la acción
}

\author{
OLBETH HANSBERG \\ Instituto de Investigaciones Filosóficas \\ UNAM (México)
}

\begin{abstract}
RESUMEN. En este artículo me ocupo de algunas características de las emociones y de sus relaciones con otras actitudes proposicionales que les permiten ocupar un lugar en el «espacio de las razones» y funcionar así como explicaciones de acciones intencionales. Examino las diferentes formas de usar los términos de emoción y las consecuencias que esto tiene para la explicación tanto de las emociones como de las acciones. Concluyo que el modelo de emociones de Davidson es demasiado restrictivo y opto por una forma de inteligibilidad menos rígida, la de la «causación razonable», que nos permite entender un estado mental o una acción como un efecto razonable de un conjunto de estados proposicionales. Finalmente menciono cómo la atribución de rasgos de la personalidad y otras actitudes generales sirven para guiar nuestras explicaciones y delimitar el tipo de estados mentales que pueden intervenir en ellas.
\end{abstract}

ABSTRACT. In this paper I shall examine some features of emotions and of their connections with other propositional attitudes like beliefs, thoughts and other pro-attitudes. Emotions have a place in «the space of reasons» and we often explain intentional actions by reference to emotions. I go through the different ways we use emotion words and the consequences this has on the explanation of emotions and of actions. Although most emotions have a propositional content, I think that Davidson's model is too restrictive as an explanation of emotion. I argue that we are also capable of understanding emotion, or action, as a «reasonable» effect of other propositional attitudes. Finally I also try to say something about the role of traits and other general attitudes, in guiding explanations and in limiting the kind of mental states that can figure in them.

En los últimos años la filosofía se ha ocupado nuevamente del tema de las emociones. Hemos aprendido a aceptar que las emociones son un elemento esencial de nuestras vidas y que es importante estudiar sus distintos aspectos desde disciplinas diversas. Este renovado interés forma parte de una revisión de nuestra concepción de muchos de los procesos y estados psicológicos y de las complejas relaciones que existen entre ellos. Así, entre otras cosas, hemos dejado de concebir a las emociones como meras sensaciones que nos suceden, como experiencias meramente subjetivas, para concederles un contenido racional que nos permite empezar a examinar las muy intrincadas relaciones que tienen con otros estados mentales como creencias, pensamientos, deseos y otras actitudes. Por ello, las emociones ocupan ahora un lugar mucho más interesante en la explicación de nuestras acciones en general y de nuestra vida moral en especial. En este artículo pretendo examinar algunas de las relaciones que 
tienen las emociones con estados mentales intencionales y con las acciones de las personas.

Un modelo común para explicar acciones y otros estados mentales es el de las explicaciones por razones. Así, cuando un agente actúa intencionalmente lo hace por una razón. Comprendemos las acciones intencionales cuando entendemos el propósito que tenía el agente al actuar, esto es, cuando entendemos sus razones. Las razones son combinaciones de creencias y actitudes favorables ${ }^{1}$ hacia cierto tipo de acción. Pero explicar una acción, bajo cierta descripción, no implica siempre mencionar el deseo y la creencia que fueron la causa inmediata de la acción, sino que muchas veces explicamos lo que alguien hizo mencionando, entre otras cosas, emociones, rasgos de carácter, intereses, gustos, hábitos, virtudes, vicios, metas y planes más generales. La estructura de cada una de estas explicaciones será distinta y de una complejidad variable, pero todas funcionan, de manera más o menos clara, como explicaciones por razones. Por otro lado, muchos de los estados mentales, entre ellos las emociones, se explican a menudo mencionando la razón o las razones de que alguien esté en ese estado. Dentro de esta línea general intentaré examinar algunas de las características de las emociones que les permiten ocupar un lugar en el «espacio de las razones».

Frecuentemente explicamos acciones mencionando emociones: decimos que María se fue porque se enojó con su amigo; que la razón de que Pedro no viniera hoy es que tiene miedo de encontrarse con ella. Cuando estas explicaciones responden a la pregunta de por qué el agente actuó como lo hizo, podemos muchas veces descubrir en ellas los rasgos esenciales de las explicaciones por razones. Por otro lado, es frecuente también explicar emociones dando razones: decimos, por ejemplo, que la razón de que Juan se enojara con su hijo es que le mintió, que la razón de que María no subiera al campanario es que tiene miedo a las alturas. Si las emociones responden a razones y funcionan como tales, es importante aclarar qué clase de estados mentales son, cómo las atribuimos a los otros y cuáles son sus relaciones con actitudes proposicionales como creencias, pensamientos, deseos, intenciones y otras actitudes favorables que en el lenguaje cotidiano mencionamos como constituyendo razones.

No podemos entonces dar cuenta de las emociones humanas sin considerarlas como parte de una red de otros estados mentales que puede incluir estados proposicionales como los ya mencionados, así como a percepciones, sentimientos, sensaciones, imágenes, etc. El carácter holista de los estados mentales hace que no puedan atribuirse aisladamente, sino que atribuir uno es

${ }^{1}$ Por actitud favorable puede entenderse cualquier actitud a favor de una acción. Esto incluye no sólo a los deseos, sino también otras actitudes tanto momentáneas como más permanentes. Desde un impulso, una inclinación, un gusto, sentir atracción o interés por algo, hasta considerar algo como un deber, como una obligación o valorarlo positivamente. También podríamos hablar de actitudes negativas o desfavorables hacia tipos de acción. 
atribuir un conjunto de ellos y su contenido dependerá, en múltiples ocasiones, del lugar que ocupa en el conjunto. Dado que los seres humanos tienen un sistema enormemente complejo de conceptos y actitudes proposicionales y un lenguaje y son, por ello, capaces de una amplísima gama de conductas, las emociones humanas adquieren características que las hacen diferentes $-\mathrm{O}$ al menos mucho más complejas- que las emociones que pudieran tener los animales no humanos ${ }^{2}$.

Lo dicho hasta ahora constituye un marco conceptual que me permite situar algunas características de las emociones sobre las que abundaré a continuación. Supondré, pues, que algunas actitudes proposicionales son causas $\mathrm{y}$ razones ${ }^{3} \mathrm{y}$, también efectos, de emociones y que las emociones muchas veces son ellas mismas actitudes proposicionales, es decir, tienen un contenido proposicional. Las actitudes proposicionales nos permiten dar cuenta de las emociones en el sentido de que son esenciales para identificar, distinguir y explicar las diversas emociones. Así, mi miedo de que el hombre que me apunta con la pistola me asalte se explica porque tengo una serie de creencias pertinentes $y$, junto con la creencia de que un asalto puede hacerme daño, también un fuerte deseo de mantener mi integridad física. $\mathrm{O}$, creer que mi amigo Juan me engañó explica mi enojo si tengo, entre otras, una actitud negativa frente a los engaños en general, frente a este en especial y además ciertas expectativas y creencias en relación a los amigos y a Juan en particular. Dada la gran diversidad de estados mentales que llamamos emociones, no creo que exista un conjunto específico de actitudes proposicionales que sean necesarias para todas y cada una de las emociones, sino que es necesario desglosar el conjunto de actitudes que son pertinentes para cada una de las emociones o para casos específicos o paradigmáticos de una emoción determinada. Como ejemplo de lo anterior podríamos mencionar que emociones como el enojo, el resentimiento y la indignación comparten un núcleo común de actitudes proposicionales, a saber, la creencia de que $p$, y una actitud negativa hacia $p$, pero intervienen también otras creencias, pensamientos y actitudes que las distinguen. Así, la indignación, a diferencia del enojo y del resentimiento, puede clasificarse como una emoción moral y, como tal, requiere de un conjunto complejo de conceptos, creencias y deseos relacionados con la moralidad. Un ser humano podría enojarse por una acción de otro porque afecta sus intereses o sentir resentimiento hacia él, sin por ello tener la capacidad de indignarse cuando ve cometer la misma clase de acción en contra de otra persona. Para poder indignarse es necesario que tenga la capacidad de reconocer una acción, digamos, como

\footnotetext{
${ }^{2}$ Me ocupare aquí sólo de las emociones humanas o, para ser más precisa, de las emociones que pueden funcionar como razones para actuar. No dudo de que a los animales o a los niños pequeños, e incluso a los humanos adultos, podamos atribuirles reacciones afectivas más primitivas que incluso pueden ser no conceptuales. Es posible que éstas tengan una influencia causal sobre el aparato conceptual y, por lo tanto, sobre las actitudes cognoscitivas y conativas.

${ }^{3}$ Cf. D. Davidson, «Actions, Reasons and Causes», Journal of Philosophy, núm. 60, 1963, reproducido en Essays on Actions and Events, Nueva York, Oxford Univ. Press, 1980.
} 
injusta o como moralmente deficiente, lo que, a su vez, requiere de la capacidad de tener conceptos morales. Otras emociones, como el orgullo y la alegría, implican una evaluación positiva de lo que uno cree que es el caso, pero el orgullo requiere, además, que el sujeto que siente orgullo sea capaz de autoevaluarse. Deberá juzgar que es digno de estima por el hecho de tener cierta propiedad ${ }^{4}$. Otras emociones de autoevaluación son el remordimiento, la vergüenza ${ }^{5}$ y la culpa, las cuales también pueden considerarse como emociones morales.

No sostengo, por supuesto, que las emociones sean sólo actitudes proposicionales, sino que reconozco que muy frecuentemente están unidas, sobre todo cuando se trata de episodios emocionales, a cambios fisiológicos ${ }^{6}$, expresiones conductuales no intencionales y a sensaciones o experiencias fenomenológicas ${ }^{7}$. Estas últimas son características sobre todo de algunas emociones (miedo, terror, ira, cólera) en ciertas circunstancias. Cuando una emoción se siente con gran intensidad puede, en muchas ocasiones, influir en las actitudes y acciones de una persona. Si alguien está en un estado de terror o de cólera, es capaz de hacer cosas que quizá no haría si la emoción fuera menos intensa. Así, un individuo puede herir a otro porque piensa que éste lo ha insultado, aun cuando, antes de encontrarse en esa situación, pensara que herir a otro es moralmente inaceptable y que los conflictos entre las personas deben resolverse por vías pacíficas. Sin embargo, en el momento crucial actúa «dominado» por la ira. Su acción es irreflexiva, el agente no decidió hacer lo que hizo, no ponderó las cosas, simplemente lo hizo. Pero su acción es intencional si la hizo por una razón y la razón es que creía que el otro lo había insultado y deseaba hacerle daño. Ésta es la explicación que tanto él como nosotros daríamos de su conducta. Cuando alguien dice que actuó «dominado» por la ira, el miedo, el amor o que alguna otra emoción lo «cegó», muchas veces no quiere decir que no sabía lo que hacía sino sólo que en ese momento

${ }^{4}$ Cf. G. Taylor, Pride, Shame and Guilt. Emotions of Self-assessment, Oxford, Clarendon Press, 1985, y D. Davidson, «Hume's Cognitive Theory of Pride», The Journal of Philosophy, vol. LXXIII, 19, nov. 4, 1976. Reproducido en Davidson, 1980.

La vergüenza no puede verse en todos los casos como una emoción moral, mientras que el remordimiento, la indignación y la culpa sí son emociones morales. Cf. O. Hansberg, «De las emociones morales», Revista de Filosofía, 3. época, vol. IX, Madrid, Universidad Complutense, 1996.

${ }^{6}$ Pienso que estudiar la fisiología de las emociones es muy importante y por supuesto sus resultados son fundamentales para un conocimiento completo de las emociones. Además, como creo que los sucesos mentales podrán describirse algún día en términos de alguna (o algunas) ciencia(s) física(s), la descripción neurofisiológica de las emociones nos dirá muchas cosas que habrá que tomar en cuenta. Mi interés en este trabajo es examinar a las emociones en tanto descritas en términos mentales, esto es, los términos con los que comúnmente hasta los científicos (cognitivos o no) explican las emociones y la conducta de los demás.

${ }^{7}$ Existen también otras actitudes proposicionales unidas a experiencias fenomenológicas. Un ejemplo son los deseos apetitivos, que son actitudes proposicionales con un carácter fenomenológico esencial, es decir, son deseos que se sienten a diferencia de otros deseos en los que no es el caso. 
no pudo hacer otra cosa, que no pudo reflexionar, no pudo tomar en cuenta otros posibles - quizá mejores- cursos de acción, sino que actuó de manera instintiva o por un impulso incontrolable ${ }^{8}$. Sin embargo, el que actuara de esta forma irreflexiva no implica que su acción no fuera intencional, sino sólo que no intervinieron otros deseos, creencias y actitudes que normalmente forman parte de sus razones para actuar.

En otros casos, en cambio, la sensación es sólo un acompañamiento que no influye en la acción, es decir, la sensación, aunque presente, no influye en las razones que una persona tiene para actuar. Sería el caso de alguien que siente un miedo intenso, pero actúa de la manera más conveniente para salir ileso de una situación de peligro en la que huir lo llevaría a una muerte segura. Otra situación distinta sería ignorar la emoción sentida y actuar por otras razones que se consideran más adecuadas. Un ejemplo sería la persona que ayuda a otra a quien detesta, porque se da cuenta que necesita ayuda y que sólo ella está en condición de dársela. Actúa por un deseo de ayudar y supera, quizá con esfuerzo, su aversión a hacerlo. Dependerá, pues, de los distintos casos el que las sensaciones características de algunas emociones sean un factor a considerar en las explicaciones de las acciones en las que intervienen emociones.

Generalmente, cuando hablan de emociones, los filósofos se limitan a los episodios emocionales de duración más o menos corta. Pero hay formas diversas de usar los términos de emoción. Si consideramos ejemplos como: "A sintió una gran congoja cuando vio al niño herido», «B está contenta de que su hijo haya pasado el examen», «C está contenta de estar sana», «D está indignada de que haya niños que mueran de hambre», «E está orgullosa de su habilidad como cirujano». En todos estos casos nuestra emoción atañe a algo en particular - una circunstancia, un logro, una cualidad, un suceso, una posesión, etc.pero en los ejemplos anteriores se subrayan aspectos diferentes: en los primeros resaltamos el aspecto de la emoción como emoción sentida; se trata de un episodio de duración limitada durante el cual el individuo experimenta, entre otras cosas, ciertas sensaciones. En cambio, cuando alguien está contenta de estar sana o indignada de que los niños se mueran de hambre, estamos poniendo énfasis en la manera positiva o negativa en que alguien valora una situación o estado y, en ese caso, decimos que sigue estando contenta o indignada aun cuando no esté pensando en ello. La experiencia fenomenológica no parece ser aquí un elemento esencial, ya que sólo en ciertos momentos y circunstancias tiene sensaciones características la persona que está orgullosa o indignada. Por ejemplo, cuando ve a un niño muriendo de hambre. Se trata aquí de una disposición a sentir una emoción en circunstancias adecuadas. Otros ejemplos de atribución de disposiciones como «Silvia siempre tiene miedo de encontrárselo en el café»; «A Juan le enfurece que lo engañen», implican también

${ }^{8}$ En caso del miedo, por ejemplo, podríamos hablar de un patrón diseñado para una protección inmediata contra fuentes relativamente primarias de daño físico. 
que en ciertas condiciones puede darse un episodio característico de la emoción. Pero esto no es siempre así: cuando digo que $A$ está orgulloso de su habilidad como cirujano, es posible que esté orgulloso y, sin embargo, no haya sentido orgullo. El énfasis estaría en el orgullo como una actitud que permite explicar que alguien haga ciertas cosas y no otras. Se trata aquí de la atribución de emociones de plazo largo y que no implican siempre que el sujeto experimente episodios emocionales intermitentes ${ }^{9}$. Así, decimos que Juan ha estado resentido con su padre desde hace veinte años, que Ana está orgullosa de ser una buena profesora o que Pedro les teme a las devaluaciones. Estas atribuciones pueden ser correctas aun cuando la persona no haya sentido nada ni se dé ninguna alteración fisiológica. La atribución de la emoción nos permite, sin embargo, explicar ciertos rasgos de la conducta intencional de una persona, por ejemplo, el que Ana se pase horas preparando sus clases, que tenga esa voluntad de claridad, ese entusiasmo contagioso; que Pedro constantemente haga operaciones financieras para protegerse de las devaluaciones, que Juan no vea nunca a su padre, etc., etc. Algunas personas dirían que el resentimiento de Juan, el orgullo de Ana y el temor de Pedro no son emociones, porque no comprenden que hablemos de emociones sin que, en circunstancias específicas, se den sensaciones características. Piensan, pues, que las emociones tienen un carácter fenomenológico esencial. Éste es un asunto discutible del que no me ocuparé aquí. Sólo quisiera señalar que es un hecho que en nuestras explicaciones cotidianas de los actos humanos atribuimos estos términos de emoción para explicar acciones que se hacen porque la persona tiene esa emoción y que quizá no haría si no la tuviera. Estas emociones generales o actitudes a las que nos referimos con términos de emoción deben distinguirse también de los rasgos de la personalidad. Así, por ejemplo, distinguimos entre tenerle miedo a algo y ser una persona miedosa, entre estar orgulloso de algo en particular y ser una persona orgullosa, entre un episodio de compasión y ser una persona compasiva ${ }^{10}$.

Si nos limitamos por ahora a los episodios emocionales, podemos conceder que muchas emociones tienen un franco contenido proposicional que nos permite distinguirlas. El contenido proposicional de una emoción nos proporciona el objeto de la emoción que, como ya se dijo, puede ser de índole diversa, pero que se describe mediante una oración. Así, no es lo mismo que Juan se sienta culpable de que engañó a su amigo a que se sienta culpable de que su hijo chocara el coche. Se pueden tener, por supuesto, también diferentes emociones frente al mismo objeto. Alguien me apunta con una pistola para robarme: tengo miedo porque estoy en peligro; estoy enojada porque me quiere

${ }^{9}$ A veces puede darse un episodio inicial que posteriormente se suprime y es posible que la emoción siga actuando de manera inconsciente para el sujeto, aunque no necesariamente para el observador.

${ }^{10}$ Las relaciones entre la atribución de la emoción y la atribución del rasgo no son claras y habría que examinarlas con mayor cuidado. 
hacer daño; siento humillación porque me va a robar; culpa porque no atendí a las advertencias. Juan puede sentirse culpable de que María perdiera su trabajo y triste y enojado por ello. Sin embargo, existen otras instancias de emociones que no parecen tener un contenido proposicional. Distinguimos, por ejemplo, entre adscripciones proposicionales de orgullo y adscripciones no proposicionales; digamos, entre «ella está orgullosa de que su hijo ganara el premio» y «ella está orgullosa de su hijo». Muchas veces es posible especificar el orgullo no proposicional en forma proposicional: esto es así cuando queda claro para la persona que está orgullosa o para la que hace la atribución, cuál es el aspecto o cualidad de un objeto o situación el que produce orgullo ${ }^{11}$. Así, si $A$ está orgullosa de su cuadro de Miró, podemos preguntarle qué acerca de su cuadro le produce orgullo y, dependiendo de su respuesta, podemos pasar de «está orgullosa de su cuadro de Miró» a «está orgullosa de que su cuadro de Miró es muy bueno», o a «está orgullosa de que todos admiren su Miró», o a «está orgullosa de que logró ahorrar para comprarse un Miró», o a «está orgullosa de que su Miró es superior a otros cuadros del mismo autor»... o, simplemente «de que tiene un Miró». Así sucede también con otras emociones. En el caso del miedo decimos que le tenemos miedo a las guerras, a las víboras o a los fantasmas. Es bastante común tenerle miedo a un objeto respecto del cual no queda claro cuál es el aspecto específico que lo hace temible para una persona, por lo que resulta problemático fijar un contenido proposicional. Esto puede deberse a razones diversas. En ocasiones la forma no proposicional resume un conjunto de miedos proposicionales que tiene una persona en relación con un objeto o situación. El contenido de estos miedos podría variar de una persona a otra, dependiendo de cuál sea el aspecto o los aspectos del objeto que le dan miedo a cada una. Así sucede, digamos, con el miedo a la enfermedad, a la muerte, a la vejez, a la guerra. Cuando explicamos alguna creencia o acción de una persona a la que le atribuimos alguno de estos miedos, tendremos que fijar el contenido proposicional de su miedo en cada caso y dadas las circunstancias. Cuando carecemos de datos más específicos, sostener que es el miedo, digamos, a la enfermedad, lo que explica que alguien haga tal o cual cosa es una forma de señalar que algunos de los deseos, creencias y otras actitudes que suelen tener las personas que le tienen miedo a la enfermedad causaron su acción, sin que tengamos que mencionar cuáles funcionaron en este caso concreto, ya sea porque no lo sabemos exactamente o porque hay muchos aspectos que habría que mencionar para explicar un caso particular. A veces, cuando alguien tiene miedo a $x$, el no expresarlo en forma proposicional puede deberse a que la persona misma no quiere saber o simplemente ignora cuál es el aspecto de $x$ que le produce miedo. En algunos casos esto podría llevar a la búsqueda,

\footnotetext{
${ }^{11}$ Ya he expresado mis dudas acerca de la posibilidad de una receta general para transformar formas no proposicionales en proposicionales. Cf. O. Hansberg, La diversidad de las emociones, Fondo de Cultura Económica, México D. F., 1996, pp. 59-66, 109.
} 
por parte del sujeto, de un contenido proposicional, bajo la idea de que identificar el contenido del miedo podría ayudar a superarlo o al menos a manejarlo de una manera más adecuada. Otras veces se trata simplemente de que el sujeto o el intérprete no han pensado o no quieren pensar suficientemente en el asunto como para identificar el contenido o los contenidos proposicionales. De hecho es muy común, y tal vez muy conveniente, que nos conformemos con explicaciones incompletas, es decir, que mencionemos emociones sin especificar su contenido proposicional como causas razonables de acciones u otras actitudes y sin poder exponer, por lo tanto, las relaciones de contenido entre ellas y la descripción de la acción; decimos cosas como: $S$ subió al decimoséptimo piso por las escaleras porque teme a los elevadores, o no quiso confiarle su problema a su madre porque está resentido con ella.

Ahora bien, hay otras emociones cuyo objeto no parece ser proposicional y habrá que dar una versión de cómo funcionan en las explicaciones de la acción. Amor y odio son ejemplos importantes: uno por lo general ama a $A$ y no ama que $p$, uno odia a $B$ y sólo a veces odia que $p$ y, sin embargo, es muy común decir que alguien hizo algo por amor o porque odia a alguien ${ }^{12}$. Éste es un asunto complejo que no tocaré aquí, pero no quiero dejar de mencionar que la cuestión de que las emociones tengan o no un contenido proposicional me parece fundamental para entender de manera cabal la estructura de las explicaciones de la acción en la que se mencionan emociones como razones de la acción.

Me permito regresar ahora a un asunto que mencioné al principio, a saber, que es necesario ${ }^{13}$ tener un conjunto de creencias y otras actitudes para que pueda darse una emoción determinada. Así, es esencial para la culpa que uno crea que es, en cierta medida, responsable de algo que uno considera moralmente reprobable. Es necesario para el enojo que uno piense que ha sucedido algo que uno considera ofensivo o dañino, para la indignación que uno considere que ha sucedido algo injusto, etc. Las emociones, a su vez, pueden tener efectos que también son actitudes proposicionales, como, por ejemplo, el deseo de venganza que frecuentemente surge cuando hay ira o rencor, el deseo de evitar un daño posible cuando se teme algo o de reparar el mal causado en el caso del remordimiento.

Un modelo conocido de explicación de emociones que muestra cómo se relacionan causalmente las actitudes proposicionales que intervienen y cómo

${ }^{12}$ Es posible que amor y odio sean términos que se refieren no a una emoción particular, sino a un complejo de emociones que llamamos «amor» o, «odio». Esto hay que distinguirlo de, por ejemplo, del deseo sexual, de la atracción o repulsión, el agrado o el disgusto, que no tienen esa complejidad. Otros estados mentales, distintos de las emociones, que no parecen tener objeto o tienen un objeto omniabarcante, son los estados de ánimo.

${ }^{13}$ No creo que podamos dar condiciones necesarias y suficientes para una emoción determinada, sino que, dado que las emociones forman una clase muy heterogénea, sólo es posible dar condiciones necesarias para algunas emociones, como el orgullo o la indignación, mientras que para otras, como el miedo, es posible darlas sólo para un conjunto de casos centrales o paradigmáticos. 
funcionan las relaciones racionales entre sus contenidos, es la versión del orgullo de Davidson, que pretende seguir la de las pasiones indirectas de Hume ${ }^{14}$. Esta explicación se aplica, sin embargo, sólo a un grupo restringido de casos. Repasemos brevemente:

Davidson sostiene que cuando una persona está orgullosa, digamos, de que tiene una casa bella, su estado mental es causado por la creencia de que él tiene una casa bella. La creencia, para causar orgullo, deberá incluir una referencia pronominal al sujeto que tiene la creencia y expresar una relación adecuada entre él y aquello de lo que está orgulloso. La creencia de que una casa es bella no causa orgullo a menos que la persona crea que es $s u$ casa, es decir, lo que causa orgullo no es una creencia acerca de un atributo de la casa, sino una creencia acerca de la persona, a saber, que ella es propietaria de una casa bella.

Ahora bien, la creencia de $A$ de que posee una casa bella no causará su orgullo si no piensa que poseer una casa bella es de alguna manera meritorio o estimable, a saber, que ella estima a las personas en cuanto tienen casas bellas y, por lo tanto, aprueba de sí misma en cuanto propietaria de una casa bella. Es importante señalar que $A$ no estima incondicionalmente a todos los que tienen casas bellas, sino que el juicio o actitud de aprobación es de carácter prima facie, esto es, se trata de un juicio condicionado: $A$ no los estima en general, sino sólo en cierto aspecto; los estima como dueños de casas bellas.

Generalizando, entonces, podemos decir que el orgullo proposicional es un estado de autoaprobación o autoestima que puede explicarse tanto causal como racionalmente mediante una creencia y una actitud general. El que Davidson sostenga que la explicación del orgullo es tanto una explicación causal como una explicación racional depende, entre otras cosas, de que identifica el estado en el que uno se encuentra cuando está orgulloso de que $p$, con tener la actitud de aprobación de sí mismo debido a $p$ y con juzgar o sostener que uno es estimable o digno de aprobación debido a $p$. Lo mismo sucede con la actitud general de aprobación que equivale, según la interpretación que hace Davidson de Hume, a un juicio general de aprobación o mérito.

La creencia específica y la actitud general explican el orgullo de dos maneras: nos proporcionan una explicación causal y nos dan las razones que tiene una persona para estar orgullosa. La especificación de los objetos proposicionales de la creencia y actitud que causan una «pasión proposicional» nos permiten dar el contenido de la emoción. Pero también podemos inferir del contenido de la emoción la naturaleza y el contenido de la actitud y la creencia que la causaron. Por lo tanto, las causas de una emoción que proporcionan su contenido proposicional la «racionalizan» en el sentido de que alguien que

${ }^{14}$ Davidson, op. cit., 1976, y D. Hume (1739-1740), Treatise on Human Nature, 1951, Selby Bigge (ed.), Oxford, Clarendon Press, en castellano Tratado de la Naturaleza Humana, traducción de Félix Duque, Madrid, Tecnos, 1988, libro II. 
conoce las causas proposicionales, entiende por qué el agente siente lo que siente $^{15}$.

La introducción de la actitud general le permite a Davidson expresar la explicación en forma de silogismo, para que el razonamiento que lleva a una persona a la conclusión de que ella es estimable en cierto respecto, sea un razonamiento deductivo. Esto implica que, si una persona es racional, podemos predecir su orgullo a partir de las premisas. La explicación que resulta es completa: una persona que tiene las actitudes pertinentes expresadas mediante un juicio universal y una creencia particular, estará orgullosa, a menos que sea imbécil o irracional, esto es, a menos que se viole o no funcione en un caso específico, alguno de los principios de racionalidad ${ }^{16}$ y no sea posible, por consiguiente, ofrecer una explicación en términos de razones.

Pero la teoría de Davidson es demasiado nítida e intelectual y funciona sólo en los casos más simples y, sobre todo, cuando el agente tiene ya de antemano la creencia universal pertinente, esto es, $A$ cree que los propietarios de casas bellas son dignos de aprobación y, al encontrarse como propietario de una bella casa, aplica esta creencia general a su caso particular ${ }^{17}$. Sin embargo, las emociones no son casi nunca resultados de silogismos. Por lo general usamos un modelo de inteligibilidad menos estricto que hace referencia a lo que sería humano y natural sentir para una persona en una circunstancia particular y dadas sus otras creencias y actitudes pertinentes. Usamos más bien la noción de «causación razonable» ${ }^{18}$. que puede expresarse diciendo que para que un estado proposicional (creencia, deseo, intención, algunas emociones, etc.) de una persona cause razonablemente otro estado proposicional, el primer estado tiene que causar el segundo y tiene que darse alguna relación de razón entre los contenidos proposicionales de ambos estados. Siempre que un conjunto de estados proposicionales causa razonablemente o «da lugar a» algún otro

${ }^{15}$ Cf. Davidson, «Reply to Bennett» en Vermazen \& Hintikka (eds.), Essays on Davidson: Actions and Events, Oxford, Oxford Univ. Press, 1985, p. 216.

${ }^{16}$ Los principios de racionalidad son aquellos principios que especifican lo que se necesita para la consistencia y la coherencia racionales. «... la satisfacción de condiciones de consistencia y coherencia racionales pueden considerarse como constitutivos del rango de aplicación de conceptos tales como creencia, deseo, intención y acción» (Davidson, 1980, p. 237). En otra parte afirma que, en la interpretación, «las relaciones entre creencias juegan un papel constitutivo decisivo; un intérprete no puede aceptar desviaciones grandes u obvias de sus propios estándares de racionalidad sin destruir el fundamento de inteligibilidad en el que se apoya toda interpretación. La posibilidad de comprender el habla o las acciones de un agente depende de la existencia de un patrón básicamente racional, un patrón que, en gran medida, deberá ser compartido por todas las criaturas racionales», Davidson, «The Structure and Content of Truth», en The Journal of Philosophy, vol. LXXXVII, núm. 6, 1990, pp. 319-20. En varios artículos, entre ellos los que acabo de mencionar, Davidson usa la teoría de las decisiones y la teoría de la elección racional para precisar nuestra comprensión de la racionalidad, pero también examina las limitaciones de dichas teorías.

${ }^{17}$ Sin embargo, en otra situación, alguien podría formarse una opinión general acerca de propietarios de casas bellas al contemplar su propia posición como propietario.

${ }^{18}$ Cf. M. Platts, Moral Realities, Londres, Routledge, 1991, pp. 57-8. 
conjunto de estados proposicionales, el reconocimiento de la causación razonable nos permite comprender por qué sucedió el efecto razonable. Hemos dado ya varios ejemplos, mencionaré otros dos: la creencia de que la acción de una persona manifiesta mala voluntad hacia otra produce resentimiento en ella; la creencia de un sujeto de que la situación en la que se encuentra es peligrosa da lugar a que tenga miedo. Al examinar estos casos podemos comprender por qué es razonable que ocurriera el efecto: lo vemos como una respuesta razonable a las circunstancias del caso. Sin embargo, entender por qué se dio ese efecto en una situación específica no implica que, cuando no sucede dicho efecto en otros casos estructuralmente similares, el agente esté transgrediendo algún principio de razón teorética o práctica. Aceptamos como razonable la relación entre los estados proposicionales implicados y también aceptamos tácitamente como razonable la relación entre los contenidos proposicionales de esos estados proposicionales. Pero, de lo anterior, no se sigue que, si no se produce el efecto, el agente es necesariamente irracional. Así, es posible que sea razonable tener miedo en una determinada situación peligrosa, pero no es necesariamente irracional no tenerlo. Cuando no se produce el efecto potencialmente razonable, se podrá ofrecer, en muchas ocasiones, una explicación de la ausencia del efecto en términos de otros aspectos de la vida mental o personal del sujeto y, dependerá de la explicación el que se le pueda atribuir o no algún elemento de irracionalidad ${ }^{19}$.

Me parece, entonces, que es posible comprender y explicar el que alguien tenga una emoción particular en circunstancias específicas, apelando a razones constituidas por un conjunto de creencias y actitudes particulares que la persona tiene en un momento dado, sin que ello requiera que, en todos los otros casos en los que concurran una situación y un conjunto de actitudes estructuralmente similares ${ }^{20}$, tenga (racionalmente) que producirse un estado emocional de cierta clase.

Lo anterior no quiere decir, por supuesto, que al interpretar la conducta no nos apoyemos, entre otras cosas, en rasgos generales, patrones recurrentes y generalizaciones más o menos vagas que guían nuestras atribuciones de estados mentales en circunstancias específicas. Lo que frecuentemente hacemos, por ejemplo, es situar las explicaciones de la conducta intencional de un individuo que mencionan sentimientos y actitudes en un contexto de relaciones sociales. El interés de la explicación se centra, no en una emoción o una acción aislada, sino en la conducta como respuesta a las acciones, actitudes y emociones de otras personas cuya conducta, a su vez, se ve como respuesta a otras acciones, actitudes y emociones nuestras y de los demás. Así, reconocer la emoción

${ }^{19}$ Platts, por ejemplo, sostiene, y yo coincido, que son posibles casos en los que pudo haber intervenido algo puramente físico, esto es, casos en los que no tenemos una explicación de la ausencia del efecto en términos de razones (op. cit., pp. 22-24).

${ }^{20}$ Es importante aquí analizar con cuidado qué deberá entenderse por «estructuralmente similares», ya que puede ser que la diferencia se vea cuando se considera un conjunto más amplio de estados mentales o rasgos generales que pueden atribuirse a una persona. 
que tiene una persona en un momento dado puede guiar mi conducta, como cuando cambio la conversación al ver que está causando turbación en el otro, o cuando trato de animarlo al ver su tristeza. Tener una idea acerca del tipo de cosas que ofenden, agradan, molestan, disgustan, indignan... a otras personas, nos permite entender por qué hacen o dejan de hacer ciertas cosas, y nos ayuda a regular nuestra propia conducta con ellas, de tal forma que podamos promover ciertas actitudes y tratar de inhibir otras. Esta capacidad ha sido ejercida por los hombres tanto para una convivencia civilizada y respetuosa como para la manipulación y sujeción inaceptables.

La referencia a emociones nos permite, pues, unificar y, por tanto, entender y explicar todo un conjunto de acciones muy variadas y aparentemente inconexas que, sin embargo, responden a un esquema de comportamiento. Ejemplo de esto son las cosas muy diferentes que podría hacer un individuo que siente resentimiento y que podrían interpretarse como manifestaciones de esta emoción; también la conducta tan diversa que podría mostrar una persona que siente remordimiento y que es posible interpretar como una expresión de su culpabilidad o como el intento de reparar el daño causado. Mencionar una emoción puede ser también una forma de unificar un gran número de deseos específicos al verlos como provenientes de un origen común en una emoción determinada. Por ejemplo, los deseos que tiene una persona de hacer cosas distintas que expresen su amor o su odio por alguien.

También nos puede guiar en nuestra interpretación saber cosas como que una persona acepta ciertas normas de conducta, tanto morales como no morales, que tiene planes a largo plazo, que reconoce obligaciones y valora ciertas cosas, que posee rasgos de carácter y habilidades específicos, que ha cultivado determinados hábitos. Inclusive las atribuciones de algo tan general y vago como el temperamento, esto es, que un individuo sea de temperamento alegre, melancólico o colérico pueden ser útiles. Recurrir a estos rasgos generales puede ayudarnos a entender por qué alguien tiende a ver una situación de un modo tal que provoca en él una emoción particular y por qué tiende a hacer ciertas cosas y no otras. También estados menos permanentes como los estados de ánimo pueden ser importantes para, en un momento dado, guiar nuestra atribución de estados mentales específicos que constituyen la explicación de una acción o un estado mental determinado.

Sin embargo, hay que entender todavía mucho mejor qué son y cómo funcionan los estados de ánimo ${ }^{21}$ y los rasgos generales como los antes mencionados no tienen un lugar claro en las explicaciones de acciones particulares. Así, mencionar rasgos de la personalidad como ser optimista, avaro, miedoso, cré-

${ }^{21}$ Los estados de ánimo, como depresión y ansiedad, a diferencia de las emociones, no parecen tener objeto o tienen un objeto omniabarcante que, en todo caso, no parece tener un contenido proposicional. Sin embargo, decir que alguien está en cierto estado de ánimo, por ejemplo, deprimido, nos da una idea del tipo de deseos y creencias que tienen las personas deprimidas y de las cosas que tienden a hacer o no hacer cuando están en ese estado. 
dulo o vanidoso, no nos dice cuáles fueron las creencias, deseos o valoraciones que causaron una emoción o una acción determinada. Uno podría ser una persona que se caracteriza en general, digamos, por ser compasiva, y que actúa, en una ocasión particular, con gran indiferencia ante el sufrimiento; o ser miedosa y no sentir miedo en una ocasión que comúnmente lo provocaría en ella, ser una persona humilde y sentir orgullo de un logro particular. Y, aun si la persona, en un caso dado, actúa o tiene alguna emoción que ejemplifica el rasgo atribuido, no se sigue que podamos deducir cuáles fueron exactamente las creencias y deseos que explicarían este caso particular. Sin embargo, aunque la atribución de estos rasgos no explica acciones u emociones particulares sí nos permite caracterizar el tipo de creencias, percepciones y deseos que tiende a tener una persona en determinadas circunstancias y nos permite entender cómo es posible que llegara a ver una situación dada como humillante, admirable, benéfica o peligrosa y a sentir una emoción en vez de otra.

Las explicaciones de las emociones son, pues, semejantes a las de otras actitudes proposicionales y a las explicaciones de la acción en el sentido de que podemos dar razones para explicar por qué una persona se siente orgullosa, indignada o arrepentida y, a la luz de sus razones, o la falta de ellas, es posible juzgar a la emoción como razonable, irracional, apropiada o inapropiada. Se abre así la posibilidad de juzgar a la emoción apelando a las razones, buenas o malas, que tenía el agente para tener la emoción y de juzgar también si la reacción emocional fue o no apropiada, dadas las circunstancias o dadas las motivaciones del agente ${ }^{22}$. Estos juicios serán, a su vez, importantes para examinar la relevancia moral de las emociones y la posibilidad de una verdadera educación de las emociones que vaya más lejos que el mero control de su expresión en la acción. Si esto es así, será posible, tal vez, que los seres humanos, si piensan bien y tienen deseos apropiados ${ }^{23}$, podrán también tener emociones razonables, esto es, emociones hacia los objetos adecuados, con la intensidad adecuada y en los momentos y las circunstancias adecuadas.

\footnotetext{
${ }^{22}$ Considero que la propiedad tiene que ver, sobre todo, con el grado de intensidad, es decir, la emoción sería inapropiada cuando es demasiado violenta o calmada para lo que merecen las circunstancias o, hablando en términos de acciones, cuando ocupa un lugar demasiado importante (poco importante) en las motivaciones y deliberaciones del agente. Sin embargo, creo que éste es un tema que deberá analizarse con más cuidado.

${ }^{23}$ Lo cual, por supuesto requiere de muchas otras cosas, entre ellas, la formación de hábitos y rasgos de carácter propicios.
} 